\title{
Heterogeneous Complex Function
}

\author{
Jicheng Tao ${ }^{1, *}$, Yun Chen ${ }^{2}$ and Xuejiao Zhao ${ }^{3}$ \\ ${ }^{1}$ Department of mathematics, China Jiliang University, 310018, Hangzhou, China \\ ${ }^{2}$ Department of mathematics, China Jiliang University, 310018, Hangzhou, China \\ ${ }^{3}$ College of Liangxin, China Jiliang University, Hangzhou 310018, Hangzhou, China
}

\begin{abstract}
In this paper, we discuss heterogeneous complex analysis function and heterogeneous harmonic function, and establish the relationship between heterogeneous complex analysis function and heterogeneous harmonic function, and then obtain heterogeneous Liouville theorem.
\end{abstract}

Keywords-heterogeneous complex analytic function; heterogeneous C.R. equation; heterogeneous cauchy integral theorem; heterogeneous cauchy integral formula; liouville theorem

\section{INTRODUCTION}

Heterogeneous complex number field and heterogeneous complex function, introduced in [1], are means that generalized complex number field and generalized complex function, ,and then authors establish the relationship between heterogeneous complex analytic function and partial differential equation. By using the property on heterogeneous complex function[2], authors introduce the definitions of integration of heterogeneous complex function, establish heterogeneous Cauchy integral theorem and Cauchy integral formula. Considering the importance of Application for partial differential equation and heterogeneous analysis in [3][4], we will discuss the boundness of heterogeneous complex analytic function and its relation with heterogeneous harmonic function.

\section{HETEROGENEOUS COMPLEX FUNCTION}

\section{A. Definition of Heterogeneous Complex Numbers Field}

We first introduce some of basic notations and terminologies, the details can be found in [1].

Definition 2.1 The heterogeneous complex numbers field $\mathrm{C}_{\mathrm{k}}$ consists, by definition $\mathrm{C}_{\mathrm{k}}=\{\mathrm{z} \mid \mathrm{z}=\mathrm{a}+\mathrm{jb}\}$ where $a, b \in R, j^{2}=-k, k>0$, and the vector operations are defined by

$$
\begin{gathered}
z=a+j b, m \in R, \tau \eta \varepsilon v, m a=m a+j m b \\
z_{1}=a_{1}+j b_{1} \text { and } z_{2}=a_{2}+j b_{2} \text {, then } \\
z_{1}+z_{2}=a_{1}+a_{2}+j\left(b_{1}+b_{2}\right)
\end{gathered}
$$

It is obvious that $C_{k}$ is a vector space.
If we give a product operation by

$$
\begin{aligned}
z_{1} & =a_{1}+j b_{1} \quad \text { and } \quad z_{2}=a_{2}+j b_{2} \quad, \quad \text { then } \\
z_{1} z_{2} & =a_{1} a_{2}-k b_{1} b_{2}+j\left(a_{1} b_{2}+a_{2} b_{1}\right),
\end{aligned}
$$

And the length of $z=a+j b$ be definited by $|z|_{k}=\sqrt{a^{2}+k b^{2}}$, and then inverse element of $z=a+j b$ be $Z^{-1}=\frac{a-j b}{|z|_{k}{ }^{2}}$, hence $C_{k}$ is a field. It is called heterogeneous complex numbers field.

Remark 2.1 When $k=1$, heterogeneous complex numbers field is the same as complex number field, hence denote $C_{1}$ is usually complex number field, denote $|z|_{1}=\sqrt{a^{2}+b^{2}}$ is the length in usually complex number field .

\section{B. Heterogeneous Complex Analytic Function}

Definition 2.2. Let $\mathrm{f}$ be function from $C_{k}$ to $C_{k}, z_{0}$ be a fix point in $\mathrm{C}_{\mathrm{k}}, \quad f$ be called differentiable function in $Z_{0}$, if the following limit exists:

$$
A=\lim _{\Delta z \rightarrow 0} \frac{\Delta W}{\Delta z}=\lim _{\Delta z \rightarrow 0} \frac{f(z)-f\left(z_{0}\right)}{z-z_{0}} \text {, and then A }
$$

be called derivative of $f(z)$ in $z_{0}$ by being denoted by $f^{\prime}\left(z_{0}\right)$.

Suppose $f$ be a function in set $\mathrm{D}, f$ be called a differentiable function in $\mathrm{D}$, if every $z \in D, f(z)$ is differentiable.

Definition 2.3 Let $f$ be function from domain $\mathrm{D}$ to $\mathrm{C}_{\mathrm{k}}$, $f$ be called an analytic function in domain $\mathrm{D}$, if $f$ be a differentiable function in $\mathrm{D}, f$ be called analysis in point $\mathrm{z}$, if $f$ be a differentiable function in some domain $\mathrm{D}$, where $z \in D$. 
Theorem 2.1 Suppose $f(z)=p(x, y)+j q(x, y)$ be a function from domain $\mathrm{D}$ and $z=x+j y$. If $f$ be a differentiable function in $\mathrm{D}$, then

(1) Partial derivative $p_{x}, p_{y}, q_{x}, q_{y}$ is exist,

(2) Heterogeneous C.-R. equation is exist for $p(x, y), q(x, y)$ at point $(x, y)$

Where heterogeneous C.-R. equation satisfy the following equation system:

$$
\frac{\partial p}{\partial x}=\frac{\partial q}{\partial y}, \quad \frac{1}{k} \frac{\partial p}{\partial y}=-\frac{\partial q}{\partial x}
$$

Theorem 2.2 Suppose $f(z)=p(x, y)+j q(x, y)$ be a function from domain $\mathrm{D}$ and $z=x+j y$, then $f$ be a differentiable function in $\mathrm{D}$,if and only if

(1) $p(x, y), q(x, y)$ differentiable function for every $(x, y)$,

(2) Heterogeneous C.-R.equation is exist for $p(x, y), q(x, y)$ at point $(x, y)$.

\section{INTEGRATION OF HETEROGENEOUS COMPLEX FUNCTION}

We first introduce the definition of integration of heterogeneous complex function and its some properties, the details can be found in [2].

\section{A. Definition of Integration of Heterogeneous Complex} Function

Definition 3.1 Let $C_{j}$ be a directing curve in heterogeneous complex number field $C_{k}$ by

$$
z=z(t) \quad(\alpha \leq t \leq \beta
$$

where $a=z(\alpha)$ is starting point and $b=z(\beta)$ is end point. Let $f(z)$ is a function along $C_{j}$, some points: $a=z_{0}, z_{1}, \ldots, z_{n-1}, z_{n}=b$ will be identified along directing curve $C_{j}$ from $a$ to $b$. A point $\xi_{t}$ be took from $Z_{t-1}$ to $Z_{t}$ in the curve $C_{j} . \quad f(z)$ be called integrability along directing curve $C_{j}$,if the following limit exists:

$$
\operatorname{Lim}_{\delta \rightarrow 0} S_{n}=\operatorname{Lim}_{\delta \rightarrow 0} \sum_{l=1}^{n} f\left(\xi_{l}\right) \Delta z_{l}
$$

Where $\Delta z_{l}=z_{l}-z_{l-1}$ and $\delta=\operatorname{maX}_{1 \leq l \leq n}\left|\Delta z_{l}\right|_{k}$, then this limit be called integration of $f(z)$ along directing curve $C_{j}$ from $a$ to $b$, by being denoted by $\int_{C_{j}} f(z) \mathrm{d} z$.

Remark 3.1 Denote $\int_{C_{1}} f(z) \mathrm{d} z$ is usually the integration on complex number field.

Theorem 3.1 Suppose $f(z)=p(x, y)+j q(x, y)$ be a continuous function along directing curve $C_{j}$ and $z=x+j y$, then $f$ be integrability along directing curve $C_{j}$ with

$$
\int_{C_{j}} f(z) \mathrm{d} z=\int_{C_{j}} p \mathrm{~d} x-k q \mathrm{~d} y+j \int_{C_{j}} q \mathrm{~d} x+p \mathrm{~d} y
$$

Theorem 3.2 Let $C_{j}$ be a directing curve in heterogeneous complex number field $C_{k}$ by

$$
C_{j}: z=z(t)=x(t)+j y(t) \quad(\alpha \leq t \leq \beta)=\zeta(\tau),
$$

where $a=z(\alpha)$ is starting point, $b=z(\beta)$ is end point, $z^{\prime}(t)=x^{\prime}(t)+j y^{\prime}(t)$ is a continuous function on $[\alpha, \beta]$ and $z^{\prime}(t) \neq 0$. Let $f[z(t)]=p[x(t), y(t)]+j q[x(t), y(t)]$ is a continuous function along $C_{j}$, then

$$
\int_{C} f(z) d z=\int_{\alpha}^{\beta} f[z(t)] z^{\prime}(t) d t
$$

Theorem 3.3 Let $C_{j}$ be a directing curve in heterogeneous complex number field $C_{k}$, the length of $C_{j}$ be $L, f(z)$ be a continuous function along directing curve $C_{j}$ and $|f(z)|_{k} \leq M$,then

$$
\left|\int_{C_{j}} f(z) \mathrm{d} z\right| \leq(1+k) L M
$$

Proof:

$\left|\int_{C_{j}} f(z) \mathrm{d} z\right|_{k} \leq \int_{C_{j}}|f(z)|_{k}|d z|_{k} \leq(1+k) \int_{C_{1}}|f(z)|_{1}|d z|_{1}$, it is very obvious to us by using the Theorem 3.2 in [5]pp:100. 


\section{B. Heterogeneous Cauchy Integral Theorem and Cauchy Integral Formula}

Theorem 3.4 Suppose $f(z)$ be an analytic function from simply connected domain $\mathrm{D}$ in $C_{k}$ and $C_{j}$ be a closed curve in $\mathrm{D}$, then $\int_{C_{j}} f(z) d z=0$.

Theorem 3.5 Suppose $f(z)$ be an analytic function from simply connected domain $\mathrm{D}$ in $C_{k}$ then

$F(z)=\int_{z_{0}}^{z} f(\xi) \mathrm{d} \xi$, be a analytic function on $z$, where $Z_{0}$ is a fix point in $\mathrm{D}$ and $z \in D$.

Theorem 3.6 Suppose the boundary of domain $\mathrm{D}$ in $C_{k}$ be closed curve $C_{j}, f(z)$ be an analytic function in domain $\mathrm{D}$ and continuous function on $\bar{D}=D+C_{j}$, then

$$
f(z)=\frac{1}{2 \pi i} \int_{C_{j}} \frac{f(\xi)}{\xi-z} \mathrm{~d} \xi,(z \in D)
$$

Theorem 3.7 Suppose the boundary of domain $\mathrm{D}$ in $C_{k}$ be closed curve $C_{j}, f(z)$ be an analytic function in domain $\mathrm{D}$ and continuous function on $\bar{D}=D+C_{j}$, then there exists each order derivative for $f(z)$ and

$$
f^{(n)}(z)=\frac{n !}{2 \pi i} \int_{C_{j}} \frac{f(\xi)}{(\xi-z)^{n+1}} \mathrm{~d} \xi,(z \in D)
$$

\section{Heterogeneous Harmonic FunCtion AND HETEROGENEOUS LIOUVILLE THEOREM}

\section{A. Heterogeneous Complex Analysis Function and} Heterogeneous Harmonic Function

In this subsection, we will establish the relationship between heterogeneous complex analysis function and heterogeneous harmonic function. $k \frac{\partial^{2} p}{\partial x^{2}}+\frac{\partial^{2} p}{\partial y^{2}}=0$,

Theorem 4.1 Suppose $f(z)=p(x, y)+j q(x, y)$ be a heterogeneous analysis function from domain $D$ and $z=x+j y$. Then

$$
k \frac{\partial^{2} p}{\partial x^{2}}+\frac{\partial^{2} p}{\partial y^{2}}=0, k \frac{\partial^{2} q}{\partial x^{2}}+\frac{\partial^{2} q}{\partial y^{2}}=0
$$

\section{Definition 4.1 Denote}

$$
H_{k}=\left\{p(x, y) \mid k \frac{\partial^{2} p}{\partial x^{2}}+\frac{\partial^{2} p}{\partial y^{2}}=0, z=x+j y \in D\right\}
$$

If $p(x, y) \in H_{k}, p$ is called heterogeneous harmonic function with coefficient $k$. Suppose $f(z)=p(x, y)+j q(x, y)$ be a heterogeneous analysis function in domain $D, q$ is called heterogeneous conjugate harmonic function of $p$.

Remark 4.1 It is obviously that $f(z)=p(x, y)+j q(x, y)$ cannot be a heterogeneous analysis function for some $p, q \in H_{k}$

Theorem 4.2 Let $p(x, y) \in H_{k}$, then there exists $q$ such that $q$ is a heterogeneous conjugate harmonic function of $p$, that is, $f(z)=p(x, y)+j q(x, y)$ be a heterogeneous analysis function in domain $D$.

Proof: $\forall z=x+j y \in D, p(x, y) \in H_{k}$ then we have $k \frac{\partial^{2} p}{\partial x^{2}}+\frac{\partial^{2} p}{\partial y^{2}}=0$, and then $\frac{\partial}{\partial x}\left(\frac{\partial p}{\partial x}\right)=\frac{\partial}{\partial x}\left(-\frac{1}{k} \frac{\partial p}{\partial y}\right)$, further, there exists a total differential $q(x, y)$ such that $d q(x, y)=\frac{\partial p}{\partial x} d y-\frac{1}{k} \frac{\partial p}{\partial y} d x$ due to mathematical analysis, hence satisfied the following heterogeneous C.-R. equation

$$
\frac{\partial p}{\partial x}=\frac{\partial q}{\partial y}, \frac{\partial q}{\partial x}=-\frac{1}{k} \frac{\partial p}{\partial y}
$$

By appropriate treatment, we can find such a $q(x, y)$, which $\frac{\partial q}{\partial y}, \frac{\partial q}{\partial x}$ are two continuous functions and above heterogeneous C.-R. equation is right . Hence $f(z)=p(x, y)+j q(x, y)$ be a heterogeneous analysis function due to theorem 2.2 .

B. Heterogeneous Cauchy Inequality and Liouville Theorem

According to theorem3.3, theorem3.7, we can obtain the following heterogeneous Cauchy inequality,

Theorem 4.3 Suppose $f(z)=p(x, y)+j q(x, y)$ be a heterogeneous analysis function from domain $\mathrm{D}$ and $z=x+j y . \forall a \in D$, denote and $\Gamma=\left\{\xi|| \xi-\left.a\right|_{k}=R\right\}$ 
Such that $\bar{\Gamma}=\left\{\xi|| \xi-\left.a\right|_{k} \leq R\right\} \subset D$, Then

$$
\left|f^{(n)}(a)\right|_{k} \leq \frac{n !(1+k)}{R^{n}} M(R)
$$

Where $M(R)=\max _{z \in \Gamma}|f(z)|_{k}$

Proof: According to theorem3.7, we have,

$$
\begin{gathered}
f^{(n)}(a)=\frac{n !}{2 \pi i} \int_{\Gamma} \frac{f(\xi)}{(\xi-a)^{n+1}} \mathrm{~d} \xi, \quad(a \in D) \\
\left|f^{(n)}(a)\right|_{k}=\left|\frac{n !}{2 \pi i} \int_{\Gamma} \frac{f(\xi)}{(\xi-a)^{n}} d \xi\right|_{k}
\end{gathered}
$$

Consider theorem 3.3, then

$$
\frac{n !}{2 \pi} \int_{C_{k}}\left|\frac{f(\xi)}{(\xi-a)^{n+1}}\right|_{k}|d \xi|_{k} \leq \frac{n !}{2 \pi} \frac{M(R)}{R^{n+1}}(1+k) R .
$$

Theorem 4.4(Liouville theorem) If $f(z)$ be a bound analytic function in heterogeneous complex number field $C_{k}$, then $f(z)$ is a hetergeneous complex constant.

Proof: $\forall z \in C_{k}$, suppose $\exists M>0$, such that $|f(z)|_{k} \leq M$, by using theorem 4.3, we have $\left|f^{\prime}(z)\right|_{k} \leq \frac{(1+k)}{R} M, \quad$ let $\quad R \rightarrow \infty \quad$, then $\left|f^{\prime}(z)\right|_{k} \rightarrow 0$, hence $f^{\prime}(z)=0$, we use the same method as complex function theory, we can obtain $f(z)=C$.

\section{ACKNOWLEDGMENT}

This research was supported by the Natural Science Foundation of Zhejiang Province (No. LY16A010009)

\section{REFERENCES}

[1] Xuejiao Zhao, Yun Chen and Jicheng Tao, Heterogeneous complex number and heterogeneous complex function,Advances in Applied Mathematics, 2017, Vol, 6, 1, pp: 69-77.

[2] Yun Chen, Xuejiao Zhao and Jicheng Tao, Integration of heterogeneous complex func-tion, Advances in Applied Mathematics, 2017, Vol. 6, 2, pp: 153-164.

[3] Allen, L., Bolker, B., Lou, Y., et al. Asymptotic profile of the steady states for an SIS Epidemic Patch Model.SIAM Journal on Applied Mathematics, 2007, Vol. 67, pp: 1283-1309.
[4] Wang, W. and Zhao, X.Q., A nonlocal and time-delayed reactiondiffusion model of dengue transmission.SIAM Journal ond Applied Mathematics, 2011,Vol. 71, pp: 147-169.

[5] Zhong yuquan, Complex function theory, Higher Education Press, Beijing,2013. 